\title{
Design-based approach to $k$-nearest neighbours technique for coupling field and remotely sensed data in forest surveys
}

\author{
Federica Baffetta ${ }^{\text {a }}$, Lorenzo Fattorini ${ }^{\text {b }}$, Sara Franceschi ${ }^{\text {b }}$, Piermaria Corona ${ }^{c, *}$ \\ a Università degli Studi di Firenze, Italy \\ ${ }^{\mathrm{b}}$ Università degli Studi di Siena, Italy \\ c Università degli Studi della Tuscia, Italy
}

\section{A R T I C L E I N F O}

\section{Article history:}

Received 23 November 2007

Received in revised form 3 June 2008

Accepted 23 June 2008

\section{Keywords:}

Remotely sensed digital imagery

Forest inventories

$k$-NN method

Design-based inference

Simulation

Case study

\begin{abstract}
A B S T R A C T
The statistical properties of the $k$-NN estimators are investigated in a design-based framework, avoiding any assumption about the population under study. The issue of coupling remotely sensed digital imagery with data arising from forest inventories conducted using probabilistic sampling schemes is considered. General results are obtained for the $k$-NN estimator at the pixel level. When averages (or totals) of forest attributes for the whole study area or sub-areas are of interest, the use of the empirical difference estimator is proposed. The estimator is shown to be approximately unbiased with a variance admitting unbiased or conservative estimators. The performance of the empirical difference estimator is evaluated by an extensive simulation study performed on several populations whose dimensions and covariate values are taken from a real case study. Samples are selected from the populations by means of simple random sampling without replacement. Comparisons with the generalized regression estimator and Horvitz-Thompson estimators are also performed. An application to a local forest inventory on a test area of central Italy is considered.
\end{abstract}

(c) 2008 Elsevier Inc. All rights reserved.

\section{Introduction}

Several techniques for assessing natural resources employ information from remotely sensed imagery and ground data. Of these methodologies, the $k$-nearest neighbours $(k-\mathrm{NN})$ technique is becoming increasingly popular (Franco-Lopez et al., 2001; Katila \& Tomppo, 2001; Maselli et al., 2005; McRoberts et al., 2002; Tomppo et al., 2002; Tomppo \& Halme, 2004). Distinctively, $k$-NN is a non-parametric, multivariate technique attractive for imputing observations or combinations of observations from sampling units to estimation or mapping units (McRoberts \& Tomppo, 2007).

The fields in which the $k$-NN method has found large numbers of applications are related to the coupling of field and satellite data for forest attribute mapping and estimation. For instance, $k$-NN-based maps are being used in Finland for timber procurement by the forest industry and for habitat analyses by ecologists (Tomppo, 2005), as well as in Sweden to improve statistics from the National Forest Inventory (Nilsson et al., 2005).

The data usually available are the digital numbers from multispectral remotely sensed imagery for all the pixels in the study area, while the values of the interest variable (forest/environmental attribute) are known only for a sample of pixels. The $k$-NN prediction of the interest variable for a specific pixel is made by a weighted

\footnotetext{
* Corresponding author.

E-mail address: piermaria.corona@unitus.it (P. Corona).
}

average of the $k$ nearest pixels (with respect to a fixed metric in the covariate space) among those included in the sample.

Notwithstanding its popularity, the statistical properties of the $k$ NN method are still not clearly delineated. At least to our knowledge, the only advanced investigations on the statistical properties of $k$-NN have been performed by Kim and Tomppo (2006) and McRoberts et al. (2007) and lie in a complete model-based setting. Hence, the properties of the resulting predictors closely depend on the validity of the adopted super-population model. Furthermore, as is customary in model-based inference, these procedures do not take advantage of the fact that field data usually arise from a probabilistic sampling scheme (see e.g. Thompson, 2002, pp. 112-113 for the issue of design ignorability).

In this paper, the statistical properties of the $k$-NN estimators are derived in a design-based framework. At least to our knowledge, this is the first published attempt to analyse $k$-NN estimators from a design-based point of view. It is worth noting that design-based inference is solely determined by the characteristics of the sampling scheme and, in contrast with model-based inference, avoids any assumption about the population under study. Differences between model-based and design-based approaches are well delineated in statistical literature (e.g. Thompson, 2002, chapter 10) as well as in forestry applications (e.g. Gregoire, 1998; Schreuder et al., 1993, chapter 6). As pointed out by Särndal et al. (1992, p. 21), the main attraction of the design-based approach is that "Design-based inference is objective, nobody can challenge that the sample was really selected according to the given sampling design. The probability 